\title{
No reliable evidence for the superiority of any particular treatment for pulpally involved primary molars
}

\author{
In children with pulpally involved primary molars what pulp treatment \\ techniques are effective in retaining the tooth and avoiding long-term \\ sequelae?
}

Nadin G, Goel BR, Yeung CA, Glenny AM. Pulp treatment for extensive decay in primary teeth (Cochrane Review). The Cochrane Library 2003; Issue 1. Oxford: Update Software

Data sources Searches for reports were made using the Cochrane Oral Health Group's Trials Register, the Cochrane Central Register of Controlled Trials, MEDLINE (January 1966-August 2002), EMbase (1980-August 2002), Science Citation Index Expanded (1981-August 2002), Social Science Citation Index (1981-August 2002), Index to Scientific and Technical Proceedings (1982-August 2002) and the System for Information on Grey Literature in Europe (August 2002). Key journals were also searched by hand. There was no restriction on the language in which papers were published.

Study selection Randomised controlled trials (RCT) or quasi-RCT were selected that compared different pulp treatment techniques for extensive decay in primary molar teeth. Primary outcomes were extractions following pulp treatment and long-term effects.

Data extraction and synthesis Data extraction and quality assessment were carried out independently and in duplicate. Authors were contacted for additional information where necessary.

Results A total of 83 studies were identified but only three were suitable for inclusion, a further nine studies meeting the inclusion criteria but having inappropriate study design or analysis. The trials included investigated pulpotomy using formocresol, ferric sulphate, electrosurgical or zinc oxide eugenol pulpectomy in symptom-free, cariously exposed teeth. There were no data describing long-term effects but data on extraction following pulp treatment was available in all three studies. In two studies, there was not a statistically significant difference between the treatments. In the other study, more teeth treated by ferric sulphate pulpotomy were extracted than by zinc oxide eugenol pulpectomy but this result must be viewed with caution.

Conclusions Based on the RCT available, there is no reliable evidence supporting the superiority of one particular treatment method for pulpally involved primary molars. No conclusions can be made as to the optimum treatment or techniques for pulpally involved primary molar teeth because of the scarcity of reliable scientific research. Highquality RCT, with appropriate units of randomisation and analysis, are needed.

\section{Commentary}

Pulpotomy is recommended in all textbooks of paediatric dentistry as the appropriate treatment for pulpally involved primary teeth. The introduction to this review states that 94000 pulpotomies in primary teeth were undertaken in general practice in England and Wales in 2002 (information taken from the Dental Practice Board Digest of Statistics 2001-02 at http://www.dpb.nhs.uk). There is a wide range and an often-confusing choice of techniques and materials available. Therefore, this review addresses an important question for all dentists who treat children. Pulpotomies are used to treat deciduous teeth in various states of disease from very early or minimal pulpal involvement to nonvital abscessed teeth. As with all systematic reviews, this one addresses focused questions. The literature was first examined regarding the success at 12 months of pulpotomies, pulpectomies and direct pulp-capping of teeth with caries involving the pulp and, secondly, pulp treatment techniques and extractions in avoiding long-term sequellae.

The review, as with all Cochrane Reviews, is of the highest methodological standard. The main reasons for exclusion from the review of trials were duration of study less than 1 year or the study being a controlled clinical trial rather than a RCT.

Eighty-two studies were identified but unfortunately only three were suitable for inclusion. The authors also discussed nine studies that met the inclusion criteria but used inappropriate methodology. The consequence of this lack of data was that the reviewers were unable to identify the optimum technique. They did conclude, however, that good success rates were reported and therefore current pulpotomy techniques appear to be appropriate treatment options. There was no information on long-term sequellae.

The only criticism of this excellent review is that, given the dearth of data, it may have been useful to have conducted a subanalysis of studies of shorter duration, such as 6 months. This would provide at least some data on which to base clinical practice as we await better evidence.

Given the disappointing number of studies included it is the "implications for research" section of the review that is the most important. The dental profession, both in primary and secondary care, needs to address the issues raised if we are to provide optimal care for our patients.

\section{Chris Deery \\ Edinburgh Dental Institute, Edinburgh, UK}

Evidence-Based Dentistry (2003) 4, 30.

doi:10.1038/sj.ebd.6400178 